TITLE:

\title{
A method for optimal identification of a stop model with input- dependent shape function
}

$\operatorname{AUTHOR}(S):$

Matsuo, T; Shimasaki, M

\section{CITATION:}

Matsuo, T ...[et al]. A method for optimal identification of a stop model with inputdependent shape function. IEEE TRANSACTIONS ON MAGNETICS 2006, 42(12): 3818-3824

\section{ISSUE DATE:}

2006-12

URL:

http://hdl.handle.net/2433/40006

\section{RIGHT:}

(c)2006 IEEE. Personal use of this material is permitted. However, permission to reprint/republish this material for advertising or promotional purposes or for creating new collective works for resale or redistribution to servers or lists, or to reuse any copyrighted component of this work in other works must be obtained from the IEEE. 


\title{
A Method for Optimal Identification of a Stop Model With Input-Dependent Shape Function
}

\author{
Tetsuji Matsuo and Masaaki Shimasaki
}

\author{
Graduate School of Engineering, Kyoto University, Kyoto 615-8510, Japan
}

\begin{abstract}
An optimal identification method is developed for an efficient hysteresis model; a stop model having an input-dependent shape function. To simplify the identification procedure, the input-dependent shape function is given by the product of a weighting function and an input-independent shape function. The optimal weighting function is determined using the least-squares method. To obtain an optimal weighting function, two methods are proposed: one uses the property of equal vertical chords; the other is based on a symmetry condition. Both methods effectively improve the representation accuracy of $B-H$ loops of silicon steel sheets using the stop model. The representation capability of the stop model is compared with that of the play model.
\end{abstract}

Index Terms-Hysteresis, identification, least-squares method, silicon steel sheet, stop model.

\section{INTRODUCTION}

$\mathbf{T}$ HE Preisach model [1]-[3] is a widely used hysteresis model that precisely describes various hysteretic characteristics for arbitrary input sequences. However, large-scale electromagnetic-field analyses of ferromagnetic materials require a more efficient hysteresis model because the Preisach model has high computational costs such as a large memory requirement and the calculation cost to obtain a magnetic field $H$ inversely from a magnetic flux density $B$ [4]. This computational cost can be reduced by using the play model [5]-[7] because of its efficiency and representation capability. The play model can be simply implemented with a small memory requirement compared with the Preisach model. Moreover, a play model with input-independent shape function has been proven mathematically equivalent [8], [7] to the classical Preisach model, while a play model with input-dependent shape function is equivalent [9] to the nonlinear Preisach model proposed by Mayergoyz [2].

The stop model [5]-[7] is another choice for efficient hysteretic electromagnetic-field analyses. This model can directly provide a hysteretic output of $H$ from an input of $B$ whereas the Preisach model usually obtains an output $B$ (or a magnetization $M$ ) from $H$. The computational cost of the stop model is as small as that of the play model. Several vector versions of stop models have been proposed [5], [10]-[12]. However, representation by a stop model with input-independent shape function is restricted by the property of equal vertical chords regardless of dc bias [13], [14]. Because of this property, the stop model cannot satisfactorily represent the hysteretic characteristics of silicon steel sheets [15]. To remove this property, an input-dependent shape function has been proposed for the stop model [16].

Reference [9] proved that a stop model with input-dependent shape function is mathematically equivalent to the nonlinear Preisach model of Mayergoyz, who proposed an identification

Digital Object Identifier 10.1109/TMAG.2006.884355 method for the nonlinear Preisach model [2]. That identification method uses the second-order reversal curves of a hysteretic property. However, it is not easy to measure all the second-order reversal curves that are required for identification.

Reference [16] introduced a simple form of the input-dependent shape function that is a product of a weighting function and an input-independent shape function. This product form allows the stop model with input-dependent shape function to be identified using a two-step procedure: 1) the determination of weighting function and 2) the identification of the stop model with input-independent shape function, in which the product form effectively improves the representation accuracy of $B-H$ loops of silicon steel sheets [16], [9]. However, it has not been discussed sufficiently how to find an optimal weighting function for identification of the stop model.

This paper proposes two methods for obtaining appropriate weighting functions for the stop model. Several weighting functions are compared to improve the representation of hysteretic property of grain-oriented and nonoriented silicon steel sheets. A comparison of the representation capability of stop and play models is also reported.

\section{SHAPE FunCTION}

A discretized form of the stop model having an input-dependent shape function [16] is given as

$$
H=S(B)=\sum_{k=1}^{N} g_{k}\left(s_{\eta k}(B), B\right)
$$

where $s_{\eta}$ is the stop hysteron operator having height $\eta, N$ is the number of stop hysterons, $\eta_{k}=k B_{S} / N, g_{k}$ is the input-dependent shape function for $s_{\eta k}$, and $B_{S}$ is the saturation magnetic flux density. $S(B)$ is assumed to become a single-valued function when $|B| \geq B_{S}$. An ordinary type stop hysteron operator is given by

$$
s_{\eta}(B)=\max \left(\min \left(B-B^{0}+s_{\eta}^{0}, \eta\right),-\eta\right)
$$

where $B^{0}$ and $s_{\eta}^{0}$ are the values of $B$ and $s_{\eta}$, respectively, at the previous time-point. Because the function $S(B)$ given by 
(1) and (2) has a hysteretic property even when $|B| \geq B_{S}$, this paper defines the operator $s_{\eta}(B)$ as (3) and (4)

$$
\begin{aligned}
s_{\eta}(B) & = \begin{cases}\max \left(\min \left(B-B^{0^{*}}+s_{\eta}^{0}, \eta\right),-\eta\right) & \left(\eta<B_{\mathrm{S}}\right) \\
B & \left(\eta=B_{\mathrm{S}}\right)\end{cases} \\
B^{0^{*}} & =\max \left(\min \left(B^{0}, B_{S}\right),-B_{S}\right) .
\end{aligned}
$$

Fig. 1 illustrates characteristics of this operator for $\eta<B_{\mathrm{S}}$.

A product form of the input-dependent shape function

$$
g_{k}(s, B)=w(B) g_{0 k}(s)
$$

was introduced in [16], where $w(B)$ is called a weighting function. Equations (1) and (5) imply that

$$
\begin{aligned}
& \frac{S(B)}{w(B)}=S_{0}(B) \\
& S_{0}(B)=\sum_{k=1}^{N} g_{0 k}\left(s_{\eta k}(B)\right)
\end{aligned}
$$

where $S_{0}(B)$ represents the stop model having an input-independent shape function $g_{0 k}$. The stop model (7) represents a parallel connection of general stop hysteron operators given by $g_{0 k}\left(s_{\eta k}(B)\right)$ [7], whereas the stop model (1) represents a parallel connection of more general hysterons $g_{k}\left(s_{\eta k}(B), B\right)$. Properties of parallel connection of general hysterons have been discussed in [5].

The identification of the stop model using (5) requires the determination of functions $w(B)$ and $g_{0 k}$. Several identification methods [7], [13], [16] for the stop model with input-independent shape function have been proposed to yield the input-independent shape function $g_{0 k}$. This paper proposes two methods for the determination of $w(B)$ using the least-squares method.

\section{WeIGHTING FunCtion DETERMINATION}

\section{A. The Property of Equal Vertical Chords Regardless of DC Bias}

Reference [9] proved a representation theorem: the properties (i) and (ii) below are necessary and sufficient conditions for a hysteretic nonlinearity to be represented by the stop model with input-independent shape function:

(i) the property of equal vertical chords regardless of dc bias;

(ii) the wiping-out property.

This paper assumes that the property (ii) holds for the ferromagnetic materials discussed here, and so focuses on the property (i).

Let $\Delta h(a, u)$ be the measured $B-H$ loop width along the $H$-direction for a back-and-forth input variation $B=u+B_{0}(-a \leq u \leq a)$, where $a\left(0 \leq a \leq B_{S}\right)$ is an amplitude and $B_{0}\left(\left|B_{0}\right| \leq\left|B_{S}-a\right|\right)$ is an arbitrary dc bias. The input $B$ is assumed to have no extremum other than $B_{0} \pm a$. Having property (i) means that the vertical chord $\Delta h$ does not depend on the dc bias $B_{0}$.

The representation theorem guarantees that if a hysteretic function satisfies property (i) [and property (ii)], this function is

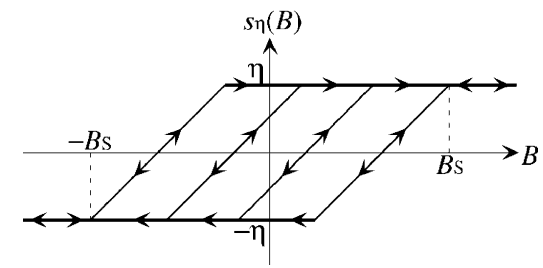

Fig. 1. Stop hysteron operator given by (3) and (4).
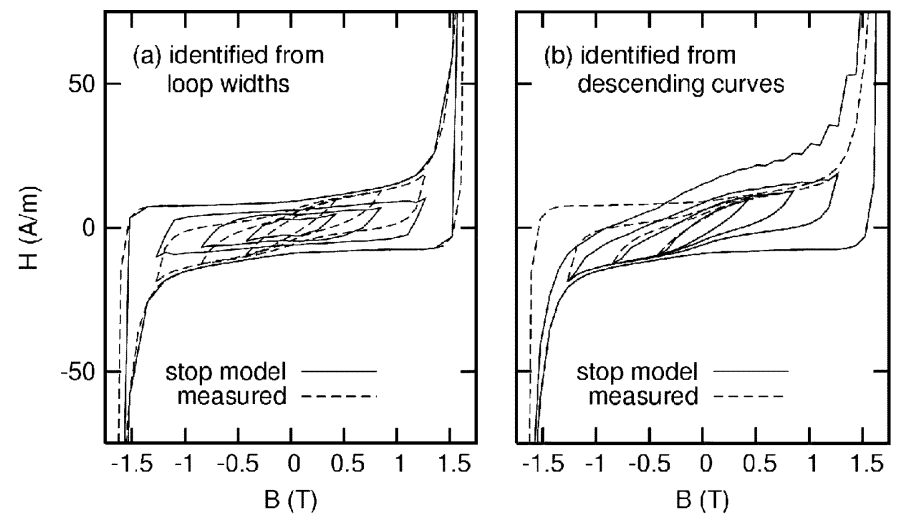

Fig. 2. $B-H$ loops of a grain-oriented silicon steel sheet represented by the stop model with input-independent shape function.

uniquely determined by $\Delta h(a, u)\left(0<a \leq B_{S}\right)$ and the major hysteresis loop.

The identification method proposed in [13] uses property (i) of the stop model with input-independent shape function. From the measured $\Delta h(a, u)$ and the major hysteresis loop, this method uniquely determines the input-independent shape function. This identification method yields a stop model that exactly represents the hysteretic property of a ferromagnetic material if this material has property (i).

Fig. 2(a) shows $B-H$ loops of a grain-oriented silicon steel sheet (JIS: 30P105) along the rolling direction simulated by the stop model with input-independent shape function, which is identified from the measured loop widths of symmetric loops. The loop widths (or vertical chords) of the simulated $B-H$ loops agree with those of the measured loops. However, the $B-H$ loops do not agree with the measured ones. This disagreement is caused by the fact that the steel sheet examined here does not have property (i). Fig. 3 compares the measured vertical chords $\Delta h_{\mathrm{S}}$ and $\Delta h_{\mathrm{A}}$ of the steel sheet that are given by (8) and (9), respectively

$$
\begin{aligned}
\Delta h_{\mathrm{S}}(a, u) & =h^{+}(a, u)-\mathrm{h}^{-}(a, u) \\
\Delta h_{\mathrm{A}}(a, u) & =h^{+}\left(a, u-B_{\mathrm{S}}+a\right)-\mathrm{h}^{-}\left(a, u-B_{\mathrm{S}}+a\right) .
\end{aligned}
$$

Therein $h^{+}(a, B)$ and $h^{-}(a, B)$ are the ascending and descending branches of the $B-H$ loop with amplitude $a$, respectively. This paper calls the $B-H$ loop consisting of $h^{ \pm}\left(a, u-B_{\mathrm{S}}+a\right)$ an "asymmetric loop" hereafter. Accordingly, $\Delta h_{\mathrm{A}}$ is the vertical chord of an asymmetric loop $\left(B_{0}=-B_{S}+a\right)$, whereas $\Delta h_{\mathrm{S}}$ is that of a symmetric loop $\left(B_{0}=0\right)$ (see Fig. 4). Vertical chords have a strong dependency on the dc bias as shown in Fig. 3, where a large discrepancy of 
vertical chords is seen between the symmetric and asymmetric loops.

\section{B. Determination of $w(B)$ Using the Property of Equal Vertical Chords}

To overcome the strong dependency that vertical chords have on dc bias, [16] introduced the weighting function $w(B)$. Equations (6) and (7) imply that $w(B)$ should be determined for $H(B) / w(B)$ to have property (i), where $H(B)$ is the measured hysteretic property. Reference [16] proposed a simple weighting function (10) using the width of a measured major $B-H$ loop.

$$
w(B)=\Delta h\left(B_{\mathrm{S}}, B\right)=h^{+}\left(B_{\mathrm{S}}, B\right)-h^{-}\left(B_{\mathrm{S}}, B\right) .
$$

The weighting function (10) reduces the dependency that vertical chords have on dc bias, but it does not completely remove the dependency.

This paper proposes an effective method to determine an optimal weighting function.

Property (i) requires that the vertical chords of symmetric loops be equal to those of asymmetric loops as (11)

$$
\frac{\Delta h_{\mathrm{S}}(a, u)}{w(u)}=\frac{\Delta h_{\mathrm{A}}(a, u)}{w\left(u-B_{\mathrm{S}}+a\right)}(-a \leq u \leq a) .
$$

Condition (11) is used to find an optimal weighting function $w(B)$. This is given by the least-squares method that determines $v_{k}(k=0, \ldots, N-1)$ to minimize

$$
\begin{aligned}
& \sum_{k=1}^{N} \sum_{m=-k+1}^{k-1}\left\{v_{|m|} \Delta h_{\mathrm{S}}\left(a_{k}, u_{m}\right)\right. \\
& \left.\quad-v_{|m+k-N|} \Delta h_{\mathrm{A}}\left(a_{k}, u_{m}\right)\right\}^{2}+\left(\sum_{k=0}^{N-1} v_{k}-1\right)^{2}
\end{aligned}
$$

where $a_{k}=k \Delta B, u_{m}=m \Delta B$ and $\Delta B=B_{S} / N$. The last term in (12) is introduced to yield a nonzero solution. A piecewise linear weighting function is given from $v_{k}$ by

$$
\begin{aligned}
w(B)= & w_{m-1}+\left(w_{m}-w_{m-1}\right) \\
& \times \frac{B-B_{m-1}}{\Delta B}\left(B_{m-1} \leq B \leq B_{m}\right) \\
w_{m}= & 1 / v_{|m|}(m=-N+1, \ldots, N-1) \\
w_{ \pm N}= & 2 w_{ \pm(N-1)}-w_{ \pm(N-2)}
\end{aligned}
$$

where $B_{m}=m \Delta B(m=-N, \ldots, N)$.

\section{Determination of $w(B)$ Based on a Symmetry Condition}

The least squares method above requires measured asymmetric $B-H$ loops, which are not always available. This subsection proposes another method that determines an optimal weighting function without needing asymmetric loops.

Reference [16] proposed another identification method of the stop model with input-independent shape function; by using the descending curves of symmetric $B-H$ loops this identification method uniquely determines the input-independent shape function. The present paper calls the identification method above "the method using descending curves" hereafter. The above method also yields a stop model that exactly represents the

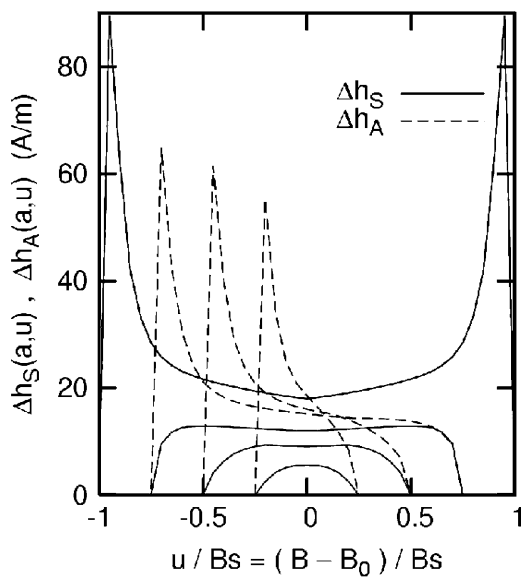

Fig. 3. Comparison of vertical chords $\Delta h_{\mathrm{S}}(a, u)$ and $\Delta h_{\mathrm{A}}(a, u)\left(a / B_{\mathrm{S}}=\right.$ $0.25,0.5,0.75,1)$

hysteretic property of a ferromagnetic material if the material has property (i).

Fig. 2(b) shows $B-H$ loops of a silicon steel sheet (JIS: 30P105) given by the stop model with input-independent shape function, which is identified by the method using descending curves. The ascending curves of the simulated $B-H$ loops are unsmooth and do not agree with the measured ones, whereas the descending curves are accurately reconstructed. This disagreement is caused by the lack of property (i) in the steel sheet, which results in an asymmetric representation of symmetric $B-H$ loops. The unsmoothness of simulated ascending curves is partly because the number of hysterons $(N)$ is small.

Most ferromagnetic materials have point-symmetric hysteretic properties with respect to the origin $((B, H)=(0,0))$. This paper assumes that the ferromagnetic materials discussed here have the point-symmetric property, and uses the symmetric property to determine an optimal weighting function.

The identification method using descending curves gives a piecewise-linear shape function as

$$
\begin{array}{r}
g_{0 k}(s)=g_{0 k}\left(s_{m-1}\right)+\kappa_{k, m-1 / 2} \frac{s-s_{m-1}}{\Delta s} \\
\left(s_{m-1} \leq s \leq s_{m}, m=-k+1, \ldots, k\right) .
\end{array}
$$

Therein $s_{m}=m \Delta s, \Delta s=B_{S} / N$, and $\kappa_{k, m-1 / 2}=g_{0 k}\left(s_{m}\right)-$ $g_{0 k}\left(s_{m-1}\right)$ given by

$$
\begin{aligned}
\kappa_{k, m-1 / 2} & =\tau_{k-1 / 2, m-1 / 2}-\tau_{k+1 / 2, m-1 / 2} \\
\tau_{k-1 / 2, m-1 / 2} & =\frac{h^{-}\left(a_{k}, B_{m}\right)}{w\left(B_{m}\right)}-\frac{h^{-}\left(a_{k-1}, B_{m-1}\right)}{w\left(B_{m-1}\right)} .
\end{aligned}
$$

If this method yields an asymmetric representation of symmetric loops, the hysteretic property $H(B) / w(B)$ cannot be exactly represented by the stop model $S_{0}(B)$, which implies that $H(B) / w(B)$ does not have property (i).

The condition for the symmetry is given by $\tau_{k-1 / 2, m-1 / 2}=$ $\tau_{k-1 / 2,-m+1 / 2}$, which means that

$$
\begin{aligned}
\frac{h^{-}\left(a_{k}, B_{m}\right)}{w\left(B_{m}\right)}- & \frac{h^{-}\left(a_{k-1}, B_{m-1}\right)}{w\left(B_{m-1}\right)} \\
& =\frac{h^{-}\left(a_{k}, B_{-m+1}\right)}{w\left(B_{-m+1}\right)}-\frac{h^{-}\left(a_{k-1}, B_{-m}\right)}{w\left(B_{-m}\right)} .
\end{aligned}
$$




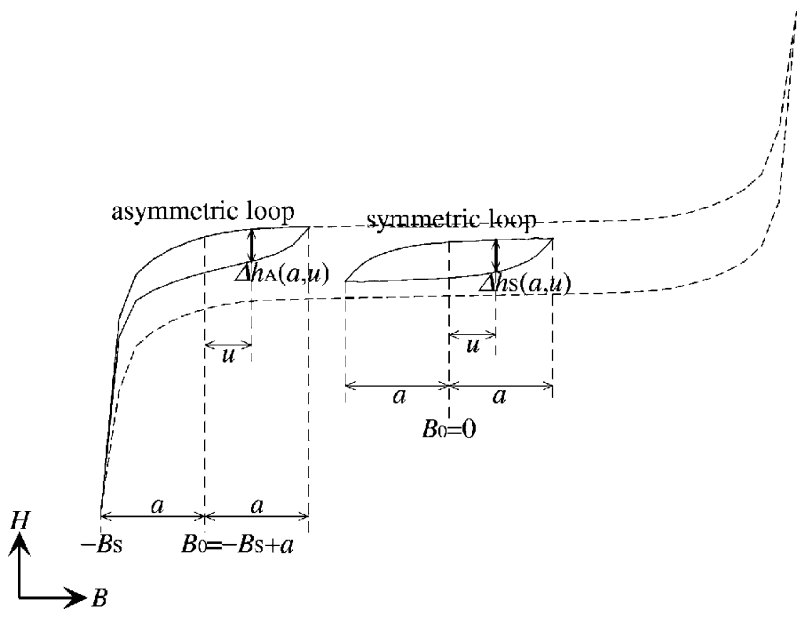

Fig. 4. Vertical chords of symmetric and asymmetric loops.
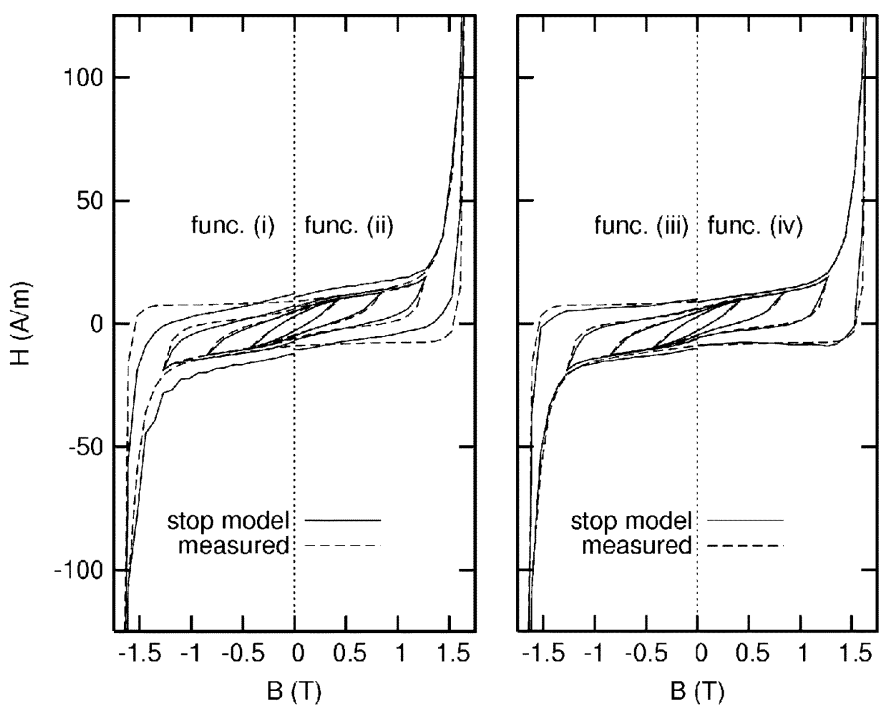

Fig. 5. Simulated symmetric $B-H$ loops of the grain-oriented silicon steel sheet along the rolling direction: (i) constant $w(B)$, (ii) major loop width, (iii) optimal $w(B)$ determined from the property of equal vertical chords, and (iv) optimal $w(B)$ determined from the symmetry condition.

From this condition, a method can be derived to obtain an appropriate weighting function. A piecewise linear weighting function (13)-(15) is given from $v_{k}(k=0, \ldots, N-1)$ that minimizes (20)

$$
\begin{aligned}
& \sum_{k=1}^{N} \sum_{m=1}^{k-1}\left[v_{m}\left\{h^{-}\left(a_{k}, B_{m}\right)+h^{-}\left(a_{k-1}, B_{-m}\right)\right\}\right. \\
& \left.\quad-v_{m-1}\left\{h^{-}\left(a_{k-1}, B_{m-1}\right)+h^{-}\left(a_{k}, B_{-m+1}\right)\right\}\right]^{2} \\
& \quad+\left(\sum_{k=0}^{N-1} v_{k}-1\right)^{2} .
\end{aligned}
$$

\section{NUMERICAL EXAMINATION}

\section{A. Simulation for a Grain-Oriented Silicon Steel Sheet}

20 symmetric and 20 asymmetric $B-H$ loops of a grain-oriented silicon steel sheet (JIS: 30P105) are measured using a single sheet tester [17], [18] at $1 \mathrm{~Hz}$ to identify the stop model
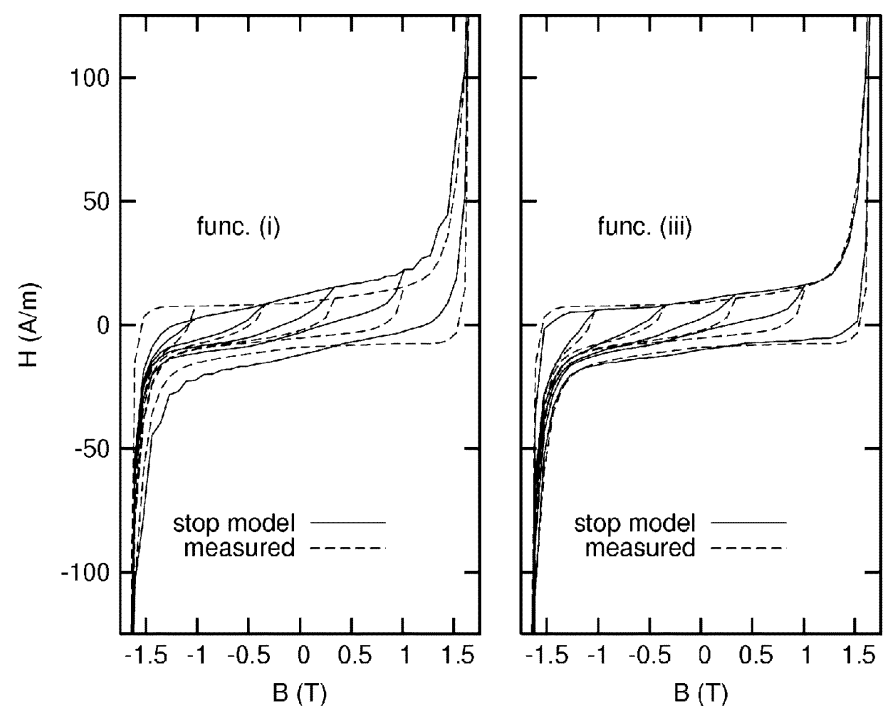

Fig. 6. Simulated asymmetric $B-H$ loops of the grain-oriented silicon steel sheet along the rolling direction in cases of functions (i) and (iii).
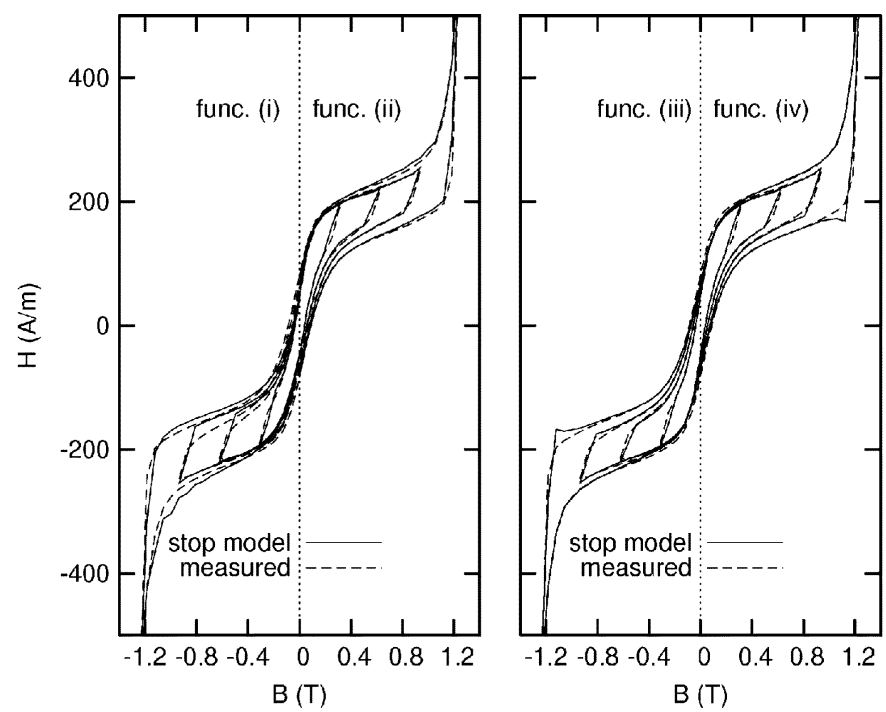

Fig. 7. Simulated symmetric $B-H$ loops of the grain-oriented silicon steel sheet along the transverse direction: (i) constant $w(B)$, (ii) major loop width, (iii) optimal $w(B)$ determined from the property of equal vertical chords, and (iv) optimal $w(B)$ determined from the symmetry condition.

with $N=20$. The following four weighting functions are examined:

(i) constant $w(B)[w(B)=1]$;

(ii) major loop width $[w(B)$ of (10)];

(iii) optimal $w(B)$ determined from the property of equal vertical chords $[w(B)$ that minimizes (12)];

(iv) optimal $w(B)$ determined from the symmetry condition [ $w(B)$ that minimizes $(20)]$.

Function (i) gives an input-independent shape function. It is shown in [16] and [9] that function (ii) improves the representation accuracy of the stop model. An identification method using the descending curves of 20 symmetric loops is used to determine $g_{0 k}$. The shape function $g_{0 k}$ is symmetrized [16] not to yield an asymmetric property as shown in Fig. 2(b).

Figs. 5 and 6 show simulated symmetric and asymmetric $B-H$ loops, respectively, of the steel sheet along the rolling 
TABLE I

AVERAGE REPRESENTATION ERRORS (\%) FOR A GRAIN-ORIENTED STEEL SHEET: (a) ROLLING DIRECTION and (b) TRANSVERSE DIRECTION

(a)

\begin{tabular}{ccccc}
\hline weighting function & (i) & (ii) & (iii) & (iv) \\
\hline \hline symmetric loops. & 1.37 & 0.97 & 0.60 & 0.57 \\
\hline asymmetric loops & 2.33 & 1.77 & 1.36 & 1.29 \\
\hline total error & 1.91 & 1.43 & 1.05 & 0.99 \\
\hline
\end{tabular}

(b)

\begin{tabular}{ccccc}
\hline weighting function & (i) & (ii) & (iii) & (iv) \\
\hline \hline symmetric loops & 2.01 & 0.97 & 0.97 & 0.92 \\
\hline asymmetric loops & 3.26 & 2.81 & 2.31 & 2.36 \\
\hline total error & 2.71 & 2.10 & 1.77 & 1.79
\end{tabular}
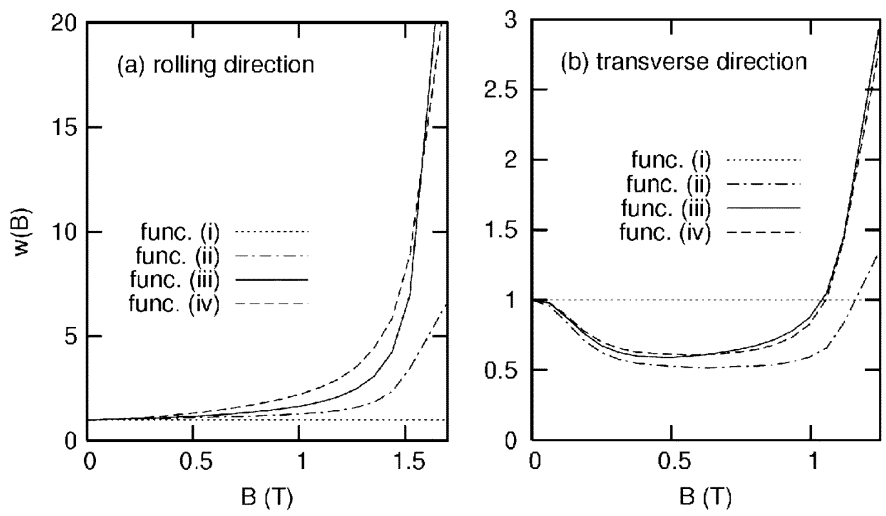

Fig. 8. Weighting functions (i)-(iv).

direction, whereas Fig. 7 shows simulated symmetric loops along the transverse direction. Table I lists the average representation errors $(\%)$ that are given as the ratio of the average discrepancy of $H$ to the maximum magnetic field. The average discrepancy is given by the root-mean-square of discrepancies between measured and simulated $H$, where the input sequence of $B$ is given by the symmetric and/or asymmetric loops (see the Appendix). The maximum magnetic fields are 277 and $612 \mathrm{~A} / \mathrm{m}$ along the rolling and transverse directions, respectively. The weighting functions (i)-(iv) are shown in Fig. 8, where the functions are normalized so that $w(0)$ is unity. Fig. 9 compares the vertical chords of the measured symmetric and asymmetric loops, $\Delta h_{\mathrm{S}} / w(B)$ and $\Delta h_{\mathrm{A}} / w(B)$, respectively, along the rolling direction in the cases of functions (iii) and (iv) (the vertical chords given by function (i) is shown in Fig. 3). The vertical chords along the transverse direction are shown in Fig. 10 for cases of functions (i) and (iii).

Figs. 5-7 and Table I show that the weighting function (iii) effectively improves the representation accuracy because it greatly reduces the dependence of the vertical chord on the dc bias, as shown in Figs. 9 and 10. Even though function (iv) is determined from only symmetric $B-H$ loops, the difference between functions (iii) and (iv) is small, as shown in Fig. 8. Function (iv) is accordingly as effective as function (iii). The smoothness of simulated $B-H$ curves is also improved by functions (iii) and (iv) without increasing $N$ as shown in Fig. 5. Figs. 5 and 7 and Table I show that function (ii) is moderately effective. However, function (ii) can be a reasonable choice because of its simplicity.
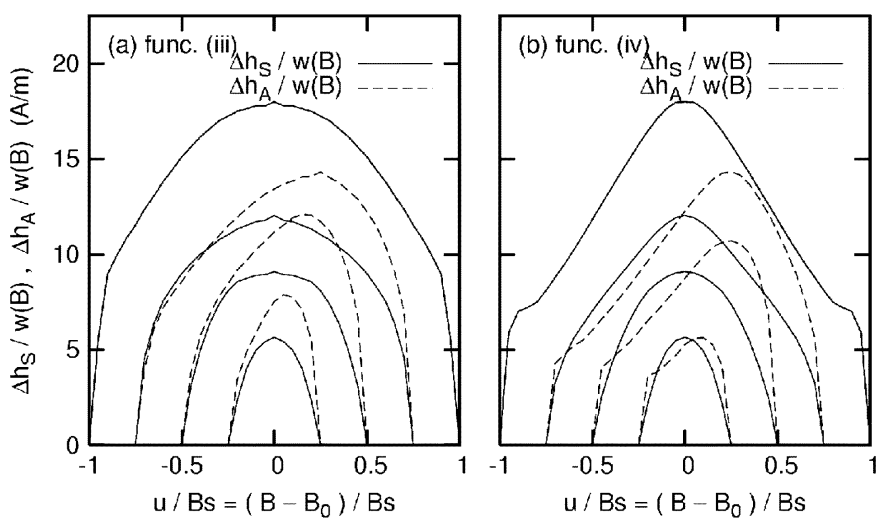

Fig. 9. Vertical chords $\Delta h_{\mathrm{S}}(a, u) / w(B)$ and $\Delta h_{\mathrm{A}}(a, u) / w(B)$ along the rolling direction in cases of functions (iii) and (iv) $\left(a / B_{\mathrm{S}}=0.25,0.5\right.$, $0.75,1)$.
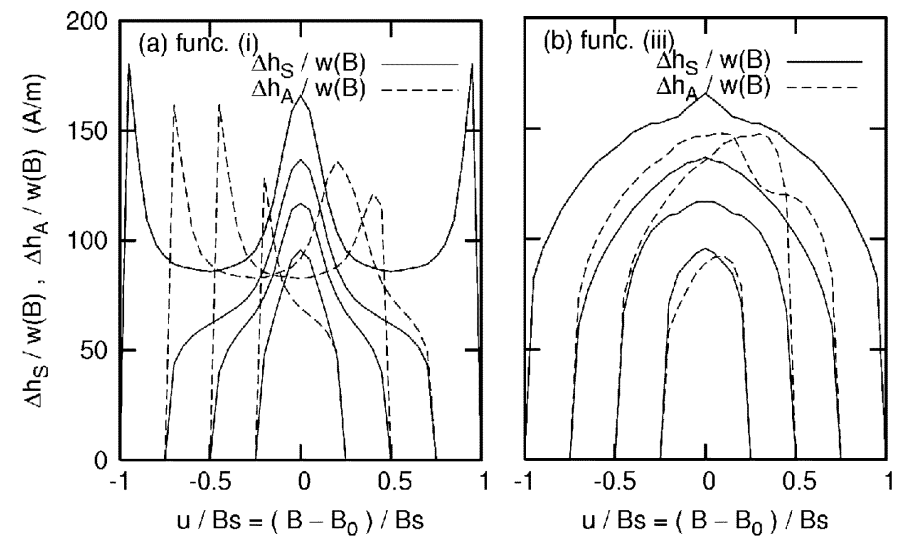

Fig. 10. Vertical chords $\Delta h_{\mathrm{S}}(a, u) / w(B)$ and $\Delta h_{\mathrm{A}}(a, u) / w(B)$ along the transverse direction in cases of functions (i) amd (iii) $\left(a / B_{\mathrm{S}}=0.25,0.5\right.$, $0.75,1)$.

\section{B. Simulation for a Nonoriented Silicon Steel Sheet}

$B-H$ loops of a nonoriented silicon steel sheet (JIS: 50A290) are represented by the stop model using the four weighting functions (i)-(iv).

Fig. 11 shows simulated symmetric $B-H$ loops of the steel sheet along the rolling direction. Table II lists the average discrepancies between measured and simulated $H$.

Fig. 11 and Table II show that among the four functions the weighting function (iii) achieves the most accurate representation. Function (iv) also effectively improves the representation accuracy without using asymmetric loops for identification.

\section{COMPARISON With Play MODEL}

An identification method of the play model with an input-dependent shape function of the product form has been proposed in [19]. Three weighting functions (i), (ii) and (iii') were examined in [19] for a grain-oriented steel sheet (JIS: 30P105). Function (iii') is the optimal weighting function proposed in [19] for the play model. This optimal function (iii'), which corresponds to function (iii) for our stop model, is determined from the congruency property of the play model by using both symmetric and asymmetric loops. 

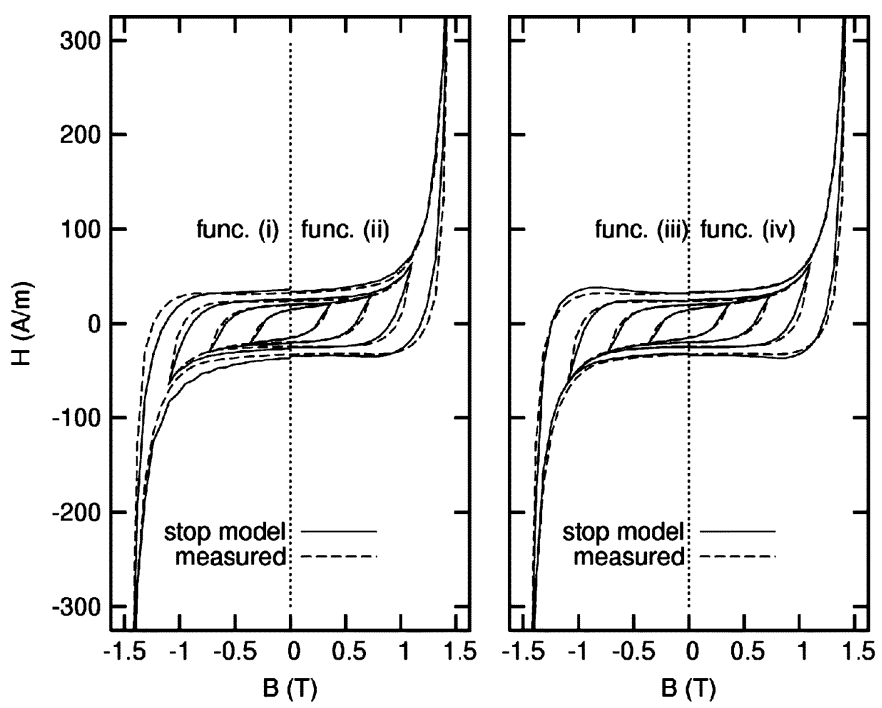

Fig. 11. Simulated $B-H$ loops of a nonoriented silicon steel sheet: (i) constant $w(B)$, (ii) major loop width, (iii) optimal $w(B)$ determined from the property of equal vertical chords, and (iv) optimal $w(B)$ determined from the symmetry condition.

TABLE II

AVERAGe REPRESENTATION ERRORS (\%) FOR A NONORIENTEd STEEL SHEET

\begin{tabular}{ccccc}
\hline weighting function & (i) & (ii) & (iii) & (iv) \\
\hline \hline symmetric loops. & 1.39 & 1.14 & 0.97 & 1.03 \\
\hline asymmetric loops & 3.39 & 2.62 & 1.47 & 2.07 \\
\hline total error & 2.59 & 2.02 & 1.25 & 1.63 \\
\hline
\end{tabular}

TABLE III

Average RePresentation ERRors (\%) By the Play Model For A GRAin-ORIENTEd STEEl SHEET. (a) ROLLING DiReCTION.

(b) TRANSVERSE DIRECTION

(a)

\begin{tabular}{cccc}
\hline weighting function & (i) & (ii) & (iii') \\
\hline \hline symmetric loops. & 0.11 & 0.11 & 0.11 \\
\hline asymmetric loops & 2.72 & 2.33 & 1.26 \\
\hline total error & 1.92 & 1.65 & 0.89 \\
\hline
\end{tabular}

(b)

\begin{tabular}{cccc}
\hline weighting function & (i) & (ii) & (iii') \\
\hline \hline symmetric loops & 0.27 & 0.27 & 0.27 \\
\hline asymmetric loops & 2.65 & 2.34 & 2.53 \\
\hline total error & 1.88 & 1.66 & 1.80 \\
\hline
\end{tabular}

The representation accuracy of the play model is compared with that of the stop model. The play model is identified from 20 symmetric $B-H$ loops with 40 play hysterons [19].

Table III lists the average discrepancies between measured and simulated $H$ of the grain-oriented steel sheet along the rolling and transverse directions. Table III shows that the play model accurately represents symmetric $B-H$ loops. Fig. 12 compares the total representation errors by stop and play models with weighting functions (i)-(iv) and (i)-(iii'), respectively. Fig. 12 shows that the stop model using (iii) or (iv) is almost as accurate as the play model using (iii'). If only symmetric $B-H$ loops are available for identification, the stop model with function (iv) achieves the smallest total representation error along the rolling direction.

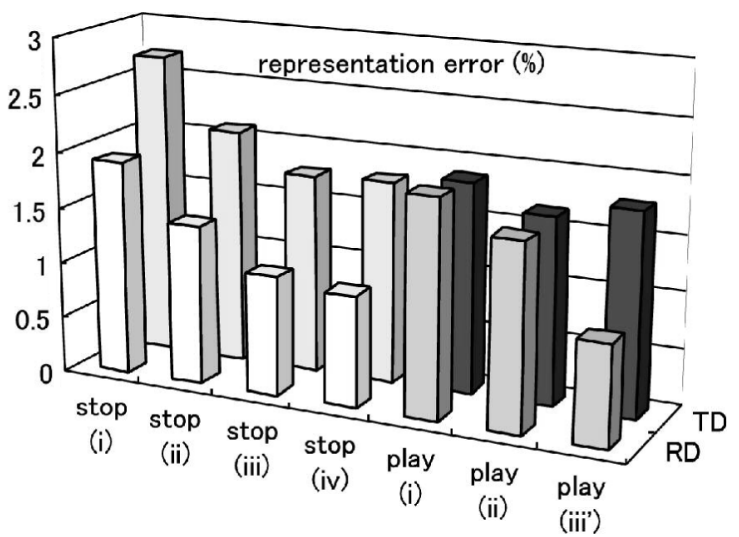

Fig. 12. Comparison of representation errors by stop and play models (RD: rolling direction, TD: transverse direction).

\section{CONCLUSION}

The two least-squares methods below are proposed to give an optimal weighting function for the stop model with an inputdependent shape function.

- The method using the property of equal vertical chords effectively improves the representation accuracy of $B-H$ loops of silicon steel sheets.

- The method based on the symmetry condition uses only symmetric $B-H$ loops, but yields a weighting function nearly as effective as the method above.

A comparison with the play model shows that the stop model using each of the weighting functions above represents the $B-H$ loops of a silicon steel sheet as accurately as the play model with an input-dependent shape function.

\section{APPENDIX}

To evaluate the representation error of symmetric loops, $N(N+2)$ points of (21) are chosen on their descending curves

$$
\begin{aligned}
(B, H(B)) & =\left(u_{\mathrm{m}}, h^{-}\left(a_{k}, u_{\mathrm{m}}\right)\right) \\
(k & =1, \ldots, N, m=-k, \ldots, k) .
\end{aligned}
$$

Therein, $a_{k}=k \Delta B, u_{m}=m \Delta B$ and $\Delta B=B_{S} / N$. To give the representation error of asymmetric loops, $N(N+2)$ points of (22) are used from their descending curves

$$
\begin{aligned}
(B, H(B)) & =\left(u_{\mathrm{m}}-B_{\mathrm{S}}+a_{k}, h^{-}\left(a_{k}, u_{\mathrm{m}}-B_{\mathrm{S}}+a_{k}\right)\right) \\
(k & =1, \ldots, N, m=-k, \ldots, k) .
\end{aligned}
$$

Both points of (21) and (22) are used to evaluate the total representation error.

\section{ACKNOWLEDGMENT}

This work was supported in part by the Japan Society for the Promotion of Science, Grant-in-Aid for Scientific Research (C), 16560243.

\section{REFERENCES}

[1] F. Preisach, "Über die Magnetische Nachwirkung," Zeitschrift für Physik, vol. 94, pp. 277-302, 1935. 
[2] I. D. Mayergoyz, Mathematical Models of Hysteresis and their Applications. New York: Springer-Verlag, 2003.

[3] E. Della Torre, Magnetic Hysteresis. Piscataway, NJ: IEEE Press, 1999.

[4] N. Takahashi, S. Miyabara, and K. Fujiwara, "Problems in practical finite element analysis using Preisach hysteresis model," IEEE Trans. Magn., vol. 35, no. 3, pp. 1243-1246, May 1999.

[5] M. A. Krasnosel'skii and A. V. Pokrovskii, Systems with Hysteresis. Berlin, Germany: Springer-Verlag, 1989.

[6] A. Visintin, Differential Models of Hysteresis. Berlin, Germany: Springer-Verlag, 1994.

[7] S. Bobbio, G. Miano, C. Serpico, and C. Visone, "Models of magnetic hysteresis based on play and stop hysterons," IEEE Trans. Magn., vol. 33, no. 6, pp. 4417-4426, Nov. 1997.

[8] M. Brokate, "Some mathematical properties of the Preisach model for hysteresis," IEEE Trans. Magn., vol. 25, no. 4, pp. 2922-2924, Jul. 1989.

[9] T. Matsuo and M. Shimasaki, "Representation theorems for stop and play models with input-dependent shape functions," IEEE Trans. Magn., vol. 41, no. 5, pp. 1548-1551, May 2005.

[10] T. Matsuo, Y. Osaka, and M. Shimasaki, "Eddy-current analysis using vector hysteresis models with play and stop hysterons," IEEE Trans. Magn., vol. 36, no. 4, pp. 1172-1177, Jul. 2000.

[11] _ - "Correction to: "Eddy-current analysis using vector hysteresis models with play and stop hysterons'," IEEE Trans. Magn., vol. 36, no. 6, pp. 4050-4051, Nov. 2000.

[12] J. V. Leite, N. Sadowski, P. Kuo-Peng, and J. P. A. Bastos, "A new anisotropic vector hysteresis model based on stop hysterons," IEEE Trans. Magn., vol. 41, pp. 1500-1503, May 2005.
[13] T. Matsuo and M. Shimasaki, "Isotropic vector hysteresis represented by superposition of stop hysteron models," IEEE Trans. Magn., vol. 37, no. 5, pp. 3357-3360, Sep. 2001.

[14] — , "Correction to: 'Isotropic vector hysteresis represented by superposition of stop hysteron models'," IEEE Trans. Magn., vol. 37, no. 6, p. 3995, Nov. 2001.

[15] T. Matsuo, D. Shimode, Y. Terada, and M. Shimasaki, "Application of stop and play models to the representation of magnetic characteristics of silicon steel sheet," IEEE Trans. Magn., vol. 39, no. 3, pp. 1361-1364, May 2003.

[16] T. Matsuo, Y. Terada, and M. Shimasaki, "Stop model with input-dependent shape function and its identification methods," IEEE Trans. Magn., vol. 40, no. 4, pp. 1776-1783, Jul. 2004.

[17] H. Nishimoto, S. Miyabara, M. Nakano, K. Fujiwara, and N. Takahashi, "Evaluation method of magnetic properties of electrical steel sheets by using the Preisach model," in Papers Technical Meeting IEE Japan, 1997, MAG-97-178.

[18] H. Nishimoto, M. Nakano, K. Fujiwara, and N. Takahashi, "Effect of frequency on magnetic properties," in Papers Technical Meeting IEE Japan, 1998, MAG-98-56.

[19] T. Matsuo and M. Shimasaki, "An identification method of play model with input-dependent shape function," IEEE Trans. Magn., vol. 41, no. 10, pp. 3112-3114, Oct. 2005.

Manuscript received March 15, 2006; revised September 11, 2006. Corresponding author: T. Matsuo (e-mail: tmatsuo@kuee.kyoto-u.ac.jp). 